\title{
MicroRNA biogenesis pathway genes polymorphisms and cancer risk: a systematic review and meta-analysis
} \author{
Qiaoyun Dai ${ }^{2}$, Xiaojin Yu ${ }^{\text {Corresp. } 1}$ \\ ${ }^{1}$ Southeast University, Department of Public Health, Nanjing, China \\ 2 National Research Institute for Family Planning, Beijing, China \\ Corresponding Author: Xiaojin Yu \\ Email address: 101006181@seu.edu.cn
}

Jieyu He ${ }^{1}$, Jun Zhao ${ }^{2}$, Wenbo Zhu ${ }^{1}$, Daxun Qi ${ }^{2}$, Lina Wang ${ }^{1}, \operatorname{Jinfang}$ Sun ${ }^{1}$, Bei Wang ${ }^{1}, \mathrm{Xu} \mathrm{Ma}^{2}$,

MicroRNAs (miRNAs) may promote the development and progression of human cancers.

Therefore, components of the miRNA biogenesis pathway may play critical roles in human cancer. Single nucleotide polymorphisms (SNPs) or mutations in genes involved in the miRNA biogenesis pathway may alter levels of gene expression, affecting disease susceptibility. Results of previous studies on genetic variants in the miRNA biogenesis pathway and cancer risk were inconsistent. Therefore, a meta-analysis is needed to assess the associations of these genetic variants with human cancer risk. We searched for relevant articles from PubMed, Web of Science, CNKI, and CBM through Jun 21, 2016. In total, 21 case-control articles met all of the inclusion criteria for the study. Significant associations were observed between cancer risk and the DGCR8 polymorphism rs417309G >A (OR 1.22, 95\% Cl 1.04-1.42), as well as the DICER1 polymorphism rs1057035T (OR 1.13, 95\% Cl 1.05-1.22). These SNPs exhibit high potential as novel diagnostic markers. Future studies with larger sample sizes and more refined analyses are needed to shed more light on these findings. 
1 MicroRNA biogenesis pathway genes polymorphisms and cancer risk: a systematic review

2

3 4

51 Department of Public Health, Southeast University, Nanjing, China;

62 National Research Institute for Family Planning, Beijing, China

$7 \quad$ \#: These authors contributed equally to this work.

26 XiaojinYu

27 E-mail: 101006181@seu.edu.cn 
28 Abstract

29

30

MicroRNAs (miRNAs) may promote the development and progression of human cancers. Therefore, components of the miRNA biogenesis pathway may play critical roles in human cancer. Single nucleotide polymorphisms (SNPs) or mutations in genes involved in the miRNA biogenesis pathway may alter levels of gene expression, affecting disease susceptibility. Results of previous studies on genetic variants in the miRNA biogenesis pathway and cancer risk were inconsistent. Therefore, a meta-analysis is needed to assess the associations of these genetic variants with human cancer risk. We searched for relevant articles from PubMed, Web of Science, CNKI, and CBM through Jun 21, 2016. In total, 21 case-control articles met all of the inclusion criteria for the study. Significant associations were observed between cancer risk and the $D G C R 8$ polymorphism rs417309G $>\mathrm{A}(\mathrm{OR} 1.22,95 \% \mathrm{CI} 1.04-1.42)$, as well as the DICER1 polymorphism rs 1057035TT (OR 1.13, 95\% CI 1.05-1.22). These SNPs exhibit high potential as novel diagnostic markers. Future studies with larger sample sizes and more refined analyses are needed to shed more light on these findings.

\section{Introduction}

MicroRNAs (miRNAs) are single-stranded RNAs of 20-30 nt that mediate posttranscriptional gene silencing. Over the past decade, it has been reported that miRNAs play key roles in the initiation and progression of human cancer For instance, the let-7 family of miRNAs can target oncogenes, such as $M Y C$ and $R A S$ family members, to inhibit tumor growth (Johnson et al. 2005; Kim et al. 2009). Hirotaka et al. (Osada \& Takahashi 2011) reported that the miR-17-92 cluster interacts with $E 2 F 1$ and $M Y C$ to promote tumor development in lung cancer. Although individual miRNAs can have either tumorigenic or tumor-suppressive functions, Jun et al. (Lu et 
51 al. 2005) analyzed 217 mammalian miRNAs from 334 samples and found that miRNAs were 52 globally down-regulated in cancers. Thomson et al. (Thomson et al. 2006) indicated that this

widespread down-regulation of miRNAs is due to a failure in a Drosha processing step, suggesting that miRNA biogenesis may be impaired in cancer.

The majority of the miRNA biogenesis pathway is shown in Fig 1. In the nucleus, microRNA genes are transcribed as primary miRNAs (pri-miRNAs) by RNA polymerase II (Pol II). These long pri-miRNAs are then cleaved by the double-stranded RNaseIII enzyme Drosha and its essential cofactor DGCR8 (Denli et al. 2004; Lee et al. 2003), generating pre-miRNAs ( 65 nt). Pre-miRNAs are then exported from the nucleus to the cytoplasm by Exportin-5 (XOP5) and its cofactor Ran (Yi et al. 2003). Following nuclear export, the pre-miRNA loop is cleaved by the RNaseIII enzyme Dicer to generate miRNA duplexes (Park et al. 2011). Dicer, TRBP or PACT, and Argonaute proteins Ago1-4 regulate the processing of pre-miRNA and the assembly of the RNA-induced silencing complex (RISC) (Chendrimada et al. 2005). Together with members of the GW182 family of proteins, one strand of the RNA duplex remains anchored to the Ago protein as the mature miRNA while the other one degraded (Gregory et al. 2005).

\section{Fig 1: miRNA biogenesis pathway.}

As mentioned in fig1, the Drosha, DGCR8, Exportin-5, Ran, and Dicer proteins are crucial components in the miRNA biogenesis pathway. Dysregulation of these miRNA-processing machinery components may disturb the product, leading to disease thus this study was focused on this five core components in miRNA biogenesis pathway and explore its link with cancer risk. Indeed, the expression levels of Drosha and Dicer are down-regulated in ovarian cancer (Kobel et al. 2009) and neuroblastomas (Lin et al. 2010), while DGCR8 is up-regulated in esophageal cancer (Sugito et al. 2006), bladder cancer (Catto et al. 2009), and prostate cancer. Exportin-5 is also down-regulated in bladder cancer (Catto et al. 2009). There are several studies implicating single- 
nucleotide polymorphisms (SNPs) present in those five miRNA biogenesis pathway genes were link with cancer risk, e.g., rs10719T $>\mathrm{C}$ in DROSHA; rs417309G $>\mathrm{A}$ and $\mathrm{rs} 1640299 \mathrm{~T}>\mathrm{G}$ in $D G C R 8 ;$ rs 11077 in $X P O 5 ; \operatorname{rs} 14035 \mathrm{C}>\mathrm{T}$ and $\mathrm{rs} 3803012 \mathrm{~A}>\mathrm{G}$ in $R A N$; and $\mathrm{rs} 1057035 \mathrm{~T}>\mathrm{C}$, rs 3742330A $>\mathrm{G}$, and rs13078T $>\mathrm{A}$ in DICER1. However, the conclusions of previous studies remain inconsistent. A comprehensive review and meta-analysis of SNPs in miRNA biogenesis pathway components is needed. In this study, a meta-analysis was conducted to evaluate the association between SNPs in five genes (DROSHA, DGCR8, XPO5, RAN, and DICERI) involved in the canonical microRNA biogenesis pathway and human cancer risk.

\section{Materials and methods}

\section{Identification of eligible studies}

Two reviewers ( $\mathrm{He}$ and $\mathrm{Zhu}$ ) searched the electronic databases PubMed, Google Scholar, CBM, and CNKI for studies containing at least one of the five gene names and its aliases (“DROSHA ETOHI2 HSA242976 RANSE3L RN3 RNASE3L RNASEN", "DGCR8 C22orf12 DGCRK6 Gy1 pahsa”, “XPO5 exp5”, "RAN ARA24 Gsp1 TC4”, or "DICER DCR1 Dicerle HERNA K12H4 8-LIKE MNG1 RMSE2"). The following terms were also used in each search: cancer, carcinoma, neoplasm, tumor, gene name and its aliases, gene polymorphism, allele, and variation. The search was limited to articles published in English or Chinese through Jun 21, 2016.The reference lists of pertinent articles were checked as well. Articles were included in the study if (1) they reported a correlation between a SNP from DROSHA (rs10719), DGCR8 (rs417309 or rs1640299), XPO5 (rs11077), RAN (rs14035), or DICER1 (rs1057035, rs3742330, or rs13078) and cancer risk; (2) they presented a case-control study; and (3) genotype frequencies were available. Articles were excluded if (1) they were review articles or focused on animals; (2) they lacked data necessary for a systematic review; or (3) the control subjects exhibited a departure 
98 from Hardy-Weinberg equilibrium (HWE). In the case where there was more than one publication

99 using the same samples, the article with the most recent publication date was selected. The two 100 reviewer reached consensus on each study.

101

102

103

104

\section{Statistical analysis}

independently and disagreement were solved by discussion: first author's name, publication year, country of origin, ethnicity, cancer types, genotype frequencies in case and control groups, HWE results, and source of control samples. Quality assessment of articles was conducted using the Newcastle-Ottawa Quality Assessment Scale (NOS) (Stang 2010). If more than one type of cancer or multistage research was involved in a single article, data were extracted as independent articles.

\section{Data extraction}

The following information were extracted by two reviewers (He and Zhao) 
121 1994; Egger et al. 1997). All $P$ value were two side and all analyses were performed by Stata

122 statistical software (Version 12.0; StataCorp, College Station, Texas USA).

123 Results

124 Study characteristics

125

126

127

128

129

130

131

132

133

134

135

136

137

138

139

140

141

142

143

144

A total of 832 relevant articles were identified based on our search strategy. Article selection procedures for all SNPs from DROSHA, DGCR8, XPO5, RAN, and DICER1 are shown in a flow chart (Fig 2). As a result, 22 case-control articles including nine SNPs (rs10719, rs417309, rs1640299, rs11077, rs14035, rs3803012, rs1057035, rs3742330, and rs13078) met the inclusion criteria for this study. Characteristics of the eligible studies are summarized in Table 1. Among the applicable articles, 15 articles were about studies of subjects who were of Asian descent, and 7 articles were about studies of subjects who were of Caucasian descent. The analyzed articles for each SNP are shown in Supplementary Table S4.

Fig 2: Flow diagram of the study selection process.

Table 1. Characteristics of the studies eligible for meta-analysis.

\section{Quantitative synthesis}

Evaluations of the associations between miRNA biogenesis pathway component SNPs and human cancer risk are shown in Table 2 and Fig 3.

Table 2. Analysis of associations between SNPs from DROSHA, DGCR8, XPO5, RAN, and DICER1 and cancer risk.

*W: major allele M: minor allele a: Asian population b: Caucasian population c: all over

\#: WM+MM vs. WW

Method: F: Fixed model R: Random model

A significant association was observed between cancer risk and the DGCR8 
$145 \mathrm{rs} 417309 \mathrm{G}>\mathrm{A}$ polymorphism in the overall pooled analysis (GA+AA vs. GG: OR 1.22, 95\% CI

146 1.04-1.42) and in the Asian samples (GA+AA vs. GG: OR 1.44, 95\% CI 1.13-1.83). For the

147 rs 1057035T $>C$ variant in DICER1, a significant (13\%) increase in cancer risk was found in the 148 overall pooled analysis (AA vs. AC+CC: OR 1.13, 95\% CI 1.05-1.22). Subgroup analysis 149 determined by sample ethnicity revealed that genotypes containing the major allele increased the 150 risk of cancer in those of Asian descent (AA vs. AC+CC: OR 1.13, 95\% CI 1.04-1.22).

\section{Sensitivity analysis}

Sensitivity analysis was conducted to assess the robustness of the results. Each

individual article was omitted to measure its effect on the pooled ORs (Fig S3). The sensitivity analysis forest plot showed that no individual article dramatically affected the pooled OR for any SNP.

\section{Publication bias}

Funnel plots did not show obvious asymmetry for any locus (Fig 4). The results of Egger's test and Begg's test revealed that rs10719 in DROSHA presented publication bias (Begg's test: $\mathrm{Z}=1.47, P=0.14$; Egger's test: $P=0.09,95 \% \mathrm{CI}-0.87-7.07)$, suggesting that the results for rs 10719 should be treated with caution. For the other SNPs, neither test indicated potential publication bias (Table 3).

Fig 4: Funnel plot for publication bias test. $\mathrm{OR}=$ odds ratio, $\mathrm{SE}=$ standard error. 
Table 3. Results of Egger's and Begg's tests for publication bias.

169

170

\section{Discussion}

In this study, we searched for articles indicating an association between SNPs in miRNA

172

biogenesis pathway genes and human cancer risk. A total of 21 articles were identified, and 9 SNPs were evaluated for association with cancer susceptibility. The results demonstrated that the GG genotype of rs417309 in DGCR8 was significantly rarer among cases compared with controls in the overall pooled analysis and the TT genotype of rs1057035 in DICERI was associated with a $13 \%$ increase in cancer risk. In addition significant associations were found in the Asian population but not in the Caucasian population, which suggested a possible ethnic difference in the genetic background and the environment. Significant association were not observed in DROSHA $(\mathrm{rs} 10719 \mathrm{~T}>\mathrm{C}), \quad X P O 5(\mathrm{rs} 11077 \mathrm{~A}>\mathrm{G})$ and $R A N(\mathrm{rs} 14035 \mathrm{C}>\mathrm{T}, \mathrm{rs} 3803012 \mathrm{~A}>\mathrm{G})$. Genome-wide association studies (GWAS) involving associations between esophageal adenocarcinoma risk and $R A N(\mathrm{rs} 14035 \mathrm{C}>\mathrm{T})$ or XOP5 (rs11077A $>\mathrm{G})$ were included in this meta-analysis (Buas et al. 2015). The results of our meta-analysis were consistent with the findings of these GWAS publications (OR 1.10, 95\% CI 0.76-1.59 compared with OR 1.17, 95\% CI 0.87-1.57 in our meta-analysis for $R A N$ rs $14035 \mathrm{C}>\mathrm{T}$ and OR $0.94,95 \%$ CI $0.85-1.04$ compared with OR $0.93,95 \%$ CI $0.87-1.01$ in our meta-analysis for $X O P 5$ rs11077A $>\mathrm{G})$.

DROSHA and its essential cofactor DGCR8 are critical protein that executes the initial step in microRNA processing (Lee et al. 2003). Dysregulation of DROSHA and DGCR8 has been observed in many cancers such as epithelial skin cancer(Sand et al. 2010), breast cancer(Chen et al. 2013) and ovarian cancer(Guo et al. 2015). Pre-miRNAs are exported into the cytoplasm to generate mature miRNAs through XOP5 and it cofactor RAN(Lin \& Gregory 2015), some heterozygous XPO5 variations were found in colon, gastric and 
191

192

193

194

195

196

197

198

199

200

201

202

203

204

205

206

207

208

209

210

211

212

213

214

endometrial cancer(Melo et al. 2010). The variation of XPO5 impairs pre-miRNA exportation and lead to defect in miRNAs biogenesis. Genetic and epigenetic association studies reported that the genetic variation of XPO5 was associated with the risk of breast cancer(Leaderer et al. 2011). DICER1 is another enzymes that plays critical role in the cleavage of pre-miRNAs into their mature form(Lin \& Gregory 2015). The variation of DICER1 were correlated with cancer risk through affecting cell proliferation and apoptosis(Dedes et al. 2011) all those component are key enzymes in miRNAs mature process. Since the global impairment of mature miRNAs is emerging as a common feature of human tumors (Melo et al. 2010), and given the critical functions of Drosha, DGCR8, Exportin-5, Ran, and Dicer in miRNA biogenesis, it is logical to presume that genetic polymorphisms in these genes may affect the processing of miRNAs and, thus, cancer risk. As a matter of fact, several studies have shown significantly association about single nucleotide polymorphism of MicroRNA biogenesis pathway gene with different cancer risk. Cho, S. H. et al.(Cho et al. 2015) reported that $R A N$ rs14035 CT heterozygotes and XPO5 rs11077AA carriers experienced reduced risk of colorectal cancer in Korean population. Leaderer, D.(Leaderer et al. 2011) performed both genetic and epigenetic association studies of XPO5 in a case-control study of breast cancer and found XPO5 rs11544382 was associated with cancer risk(the analysis of XPO5 rs11544382 with cancer risk was not performed due to lack of availed data). Chen, J. et al.(Chen et al. 2013) analyzed 1486 cervical cases and 1549 cancer-free controls in Chinese population and found that a single nucleotide polymorphisms in DICER rs1057035 and RAN rs3803012 was associated with cervical cancer risk. A case-control and further research conducted by Jiang Yue et al.(Jiang et al. 2013) suggested that DGCR8 rs417309G $>$ A might affect breast cancer risk through interrupting the miRNA binding. However, conclusions of miRNA biogenesis pathway genes polymorphisms and cancer risk remain inconsistent, which may cause by the heterogeneity of the cancer subtype, sample size and the ethnicity of patients. Yu, Y. Y(Yu et al. 2015) conducted a meta-analysis synthesizes sevens 
215 studies with multi-type cancer and found the $\mathrm{C}$ allele of the DICER rs1057035 polymorphism

216 probably decreases cancer risk(We included more studies in our studies and found the same results

217 in DICER rs1057035). This study based on the association of a single SNP or gene with cancer

218 risk, which may weaken gene's biological value. All five miRNA biogenesis pathway were

219 evaluated in our analysis, which may provide a more comprehensive view of the true system. To

220 the best of our knowledge, this study represents the first meta-analysis to focus on the association

221 between human cancer risk and SNPs across the entire miRNAs biogenesis pathway.

Sensitivity analysis indicated that the results of our study were robust. Egger's test and

Begg's test revealed that publication bias has little influence on the results, with the exception of rs 10719 in DROSHA, suggesting that the results for rs10719 should be considered with caution.

Despite these results, some limitations still apply to this meta-analysis. First, all results were based on unadjusted estimates because few adjusted ORs were reported in the included articles. Biases caused by confounding variables such as sex, age, and smoking may therefore persist. Secondly, heterogeneity among different cancers may cause real effects to be hidden when pooling all cancer types. A stratified analysis based on cancer type was not performed due to the lack of an adequate number of publications for a single cancer type. Third, although the results of the publication bias analysis indicated no bias for any locus except rs10719, it is possible that there actually was publication bias but that it was not identified by the Egger's and Begg's tests due to the low power given to the small number of studies ${ }^{[20,21]}$. Finally, we applied a traditional fixed model and a random model to pooled multiple-ethnicity samples; neither of these approaches is ideal for capturing the heterogeneity of effects observed across different ethnic populations ${ }^{[46]}$. 
238 thus potentially be used as biomarkers for cancer diagnosis. However, future well-designed

239 studies, including those focused on a single cancer type with larger sample sizes, are still needed

240 to confirm these observed associations due to the limitations mentioned above.

241

242

243

244

245

246

247

248

249

250

251

252

253

254

255

256

257

258

259

260

261

262

263

264

265

266

267

268

269

270

271

272

273

274

275

276

277

278

Begg CB, and Mazumdar M. 1994. Operating characteristics of a rank correlation test for publication bias. Biometrics 50:1088-1101.

Buas MF, Onstad L, Levine DM, Risch HA, Chow WH, Liu G, Fitzgerald RC, Bernstein L, Ye W, Bird NC, Romero Y, Casson AG, Corley DA, Shaheen NJ, Wu AH, Gammon MD, Reid BJ, Hardie L, Peters U, Whiteman DC, and Vaughan TL. 2015. MiRNA-Related SNPs and Risk of Esophageal Adenocarcinoma and Barrett's Esophagus: Post Genome-Wide Association Analysis in the BEACON Consortium. PLoS One 10:e0128617. 10.1371/journal.pone.0128617

Catto JW, Miah S, Owen HC, Bryant H, Myers K, Dudziec E, Larre S, Milo M, Rehman I, Rosario DJ, Di Martino E, Knowles MA, Meuth M, Harris AL, and Hamdy FC. 2009. Distinct microRNA alterations characterize highand low-grade bladder cancer. Cancer Res 69:8472-8481. 10.1158/0008-5472.CAN-09-0744

Chen J, Qin Z, Pan S, Jiang J, Liu L, Liu J, Chen X, Hu Z, and Shen H. 2013. Genetic variants in RAN, DICER and HIWI of microRNA biogenesis genes and risk of cervical carcinoma in a Chinese population. Chin J Cancer Res 25:565571. 10.3978/j.issn.1000-9604.2013.10.03

Chendrimada TP, Gregory RI, Kumaraswamy E, Norman J, Cooch N, Nishikura K, and Shiekhattar R. 2005. TRBP recruits the Dicer complex to Ago2 for microRNA processing and gene silencing. Nature 436:740-744. 10.1038/nature03868

Cho SH, Ko JJ, Kim JO, Jeon YJ, Yoo JK, Oh J, Oh D, Kim JW, and Kim NK. 2015. 3'-UTR Polymorphisms in the MiRNA Machinery Genes DROSHA, DICER1, RAN, and XPO5 Are Associated with Colorectal Cancer Risk in a Korean Population. PLoS One 10:e0131125. 10.1371/journal.pone.0131125

Dedes KJ, Natrajan R, Lambros MB, Geyer FC, Lopez-Garcia MA, Savage K, Jones RL, and Reis-Filho JS. 2011. Downregulation of the miRNA master regulators Drosha and Dicer is associated with specific subgroups of breast cancer. Eur J Cancer 47:138-150. 10.1016/j.ejca.2010.08.007

Denli AM, Tops BB, Plasterk RH, Ketting RF, and Hannon GJ. 2004. Processing of primary microRNAs by the Microprocessor complex. Nature 432:231-235. 10.1038/nature03049

Dersimonian R, and Nan L. 1986. Meta-analysis in clinical trials. Controlled Clinical Trials 7:177-188.

Egger M, Davey Smith G, Schneider M, and Minder C. 1997. Bias in meta-analysis detected by a simple, graphical test. BMJ 315:629-634.

Gregory RI, Chendrimada TP, Cooch N, and Shiekhattar R. 2005. Human RISC couples microRNA biogenesis and posttranscriptional gene silencing. Cell 123:631-640. 10.1016/j.cell.2005.10.022

Guo Y, Tian P, Yang C, Liang Z, Li M, Sims M, Lu L, Zhang Z, Li H, Pfeffer LM, and Yue J. 2015. Silencing the doublestranded RNA binding protein DGCR8 inhibits ovarian cancer cell proliferation, migration, and invasion. Pharm Res 32:769-778. 10.1007/s11095-013-1219-9

Gutierrez-Camino A, Lopez-Lopez E, Martin-Guerrero I, Pinan MA, Garcia-Miguel P, Sanchez-Toledo J, Carbone 
Baneres A, Uriz J, Navajas A, and Garcia-Orad A. 2014. Noncoding RNA-related polymorphisms in pediatric acute lymphoblastic leukemia susceptibility. Pediatr Res 75:767-773. 10.1038/pr.2014.43

Higgins JPT, and Thompson SG. 2002. Quantifying heterogeneity in a meta-analysis. Statistics in Medicine 21:15391558.

Horikawa Y, Wood CG, Yang H, Zhao H, Ye Y, Gu J, Lin J, Habuchi T, and Wu X. 2008. Single nucleotide polymorphisms of microRNA machinery genes modify the risk of renal cell carcinoma. Clin Cancer Res 14:7956-7962. 10.1158/1078-0432.CCR-08-1199

Jiang Y, Chen J, Wu J, Hu Z, Qin Z, Liu Xa, Guan X, Wang Y, Han J, and Jiang T. 2013. Evaluation of genetic variants in microRNA biosynthesis genes and risk of breast cancer in Chinese women. International Journal of Cancer 133:2216-2224.

Johnson SM, Grosshans H, Shingara J, Byrom M, Jarvis R, Cheng A, Labourier E, Reinert KL, Brown D, and Slack FJ. 2005. RAS is regulated by the let-7 microRNA family. Cell 120:635-647. 10.1016/j.cell.2005.01.014

Kim HH, Kuwano Y, Srikantan S, Lee EK, Martindale JL, and Gorospe M. 2009. HuR recruits let-7/RISC to repress CMyc expression. Genes Dev 23:1743-1748. 10.1101/gad.1812509

Kim JS, Choi YY, Jin G, Kang HG, Choi JE, Jeon HS, Lee WK, Kim DS, Kim CH, Kim YJ, Son JW, Jung TH, and Park JY. 2010. Association of a common AGO1 variant with lung cancer risk: a two-stage case-control study. Mol Carcinog 49:913-921. 10.1002/mc.20672

Kobel M, Gilks CB, and Huntsman DG. 2009. Dicer and Drosha in ovarian cancer. N Engl J Med 360:1150-1151; author reply 1151. 10.1056/NEJMc090085

Leaderer D, Hoffman AE, Zheng T, Fu A, Weidhaas J, Paranjape T, and Zhu Y. 2011. Genetic and epigenetic association studies suggest a role of microRNA biogenesis gene exportin-5 (XPO5) in breast tumorigenesis. Int J Mol Epidemiol Genet 2:9-18.

Lee Y, Ahn C, Han J, Choi H, Kim J, Yim J, Lee J, Provost P, Radmark O, Kim S, and Kim VN. 2003. The nuclear RNase III Drosha initiates microRNA processing. Nature 425:415-419. 10.1038/nature01957

Lin RJ, Lin YC, Chen J, Kuo HH, Chen YY, Diccianni MB, London WB, Chang CH, and Yu AL. 2010. microRNA signature and expression of Dicer and Drosha can predict prognosis and delineate risk groups in neuroblastoma. Cancer Res 70:7841-7850. 10.1158/0008-5472.CAN-10-0970

Lin S, and Gregory RI. 2015. MicroRNA biogenesis pathways in cancer. Nat Rev Cancer 15:321-333. 10.1038/nrc3932

Liu L, An J, Liu J, Wen J, Zhai X, Liu Y, Pan S, Jiang J, Wen Y, Liu Z, Zhang Y, Chen J, Xing J, Ji G, Shen H, Hu Z, and Fan Z. 2013. Potentially functional genetic variants in microRNA processing genes and risk of HBV-related hepatocellular carcinoma. Mol Carcinog 52 Suppl 1:E148-154. 10.1002/mc.22062

Lu J, Getz G, Miska EA, Alvarez-Saavedra E, Lamb J, Peck D, Sweet-Cordero A, Ebert BL, Mak RH, Ferrando AA, Downing JR, Jacks T, Horvitz HR, and Golub TR. 2005. MicroRNA expression profiles classify human cancers. Nature 435:834-838. 10.1038/nature03702

Ma H, Yuan H, Yuan Z, Yu C, Wang R, Jiang Y, Hu Z, Shen H, and Chen N. 2012. Genetic variations in key microRNA processing genes and risk of head and neck cancer: a case-control study in Chinese population. PLoS One 7:e47544. 10.1371/journal.pone.0047544

Mantel N, and Haenszel W. 1959. Statistical aspects of the analysis of data from retrospective studies of disease. $J$ Natl Cancer Inst 22:639-640.

Martin-Guerrero I, Gutierrez-Camino A, Lopez-Lopez E, Bilbao-Aldaiturriaga N, Pombar-Gomez M, Ardanaz M, and Garcia-Orad A. 2015. Genetic Variants in MiRNA Processing Genes and Pre-MiRNAs Are Associated with the Risk of Chronic Lymphocytic Leukemia. PLoS One 10:e0118905. 10.1371/journal.pone.0118905

Melo SA, Moutinho C, Ropero S, Calin GA, Rossi S, Spizzo R, Fernandez AF, Davalos V, Villanueva A, Montoya G, Yamamoto H, Schwartz S, Jr., and Esteller M. 2010. A genetic defect in exportin-5 traps precursor microRNAs in the nucleus of cancer cells. Cancer Cell 18:303-315. 10.1016/j.ccr.2010.09.007

Osada H, and Takahashi T. 2011. let-7 and miR-17-92: small-sized major players in lung cancer development. Cancer Sci 102:9-17. 10.1111/j.1349-7006.2010.01707.x

Park JE, Heo I, Tian Y, Simanshu DK, Chang H, Jee D, Patel DJ, and Kim VN. 2011. Dicer recognizes the 5' end of RNA for efficient and accurate processing. Nature 475:201-205. 10.1038/nature10198

Roy R, De Sarkar N, Ghose S, Paul RR, Pal M, Bhattacharya C, Chowdhury SKR, Ghosh S, and Roy B. 2014. Genetic variations at microRNA and processing genes and risk of oral cancer. Tumor Biology 35:3409-3414.

Sand M, Gambichler T, Skrygan M, Sand D, Scola N, Altmeyer P, and Bechara FG. 2010. Expression levels of the microRNA processing enzymes Drosha and dicer in epithelial skin cancer. Cancer Invest 28:649-653. 


\title{
$10.3109 / 07357901003630918$
}

Slaby O, Sachlova M, Brezkova V, Hezova R, Kovarikova A, Bischofova S, Sevcikova S, Bienertova-Vasku J, Vasku A, Svoboda M, and Vyzula R. 2013. Identification of microRNAs regulated by isothiocyanates and association of polymorphisms inside their target sites with risk of sporadic colorectal cancer. Nutr Cancer 65:247-254. 10.1080/01635581.2013.756530

Stang A. 2010. Critical evaluation of the Newcastle-Ottawa scale for the assessment of the quality of nonrandomized studies in meta-analyses. Eur J Epidemiol 25:603-605. 10.1007/s10654-010-9491-z

Sugito N, Ishiguro H, Kuwabara Y, Kimura M, Mitsui A, Kurehara H, Ando T, Mori R, Takashima N, Ogawa R, and Fujii Y. 2006. RNASEN regulates cell proliferation and affects survival in esophageal cancer patients. Clin Cancer Res 12:7322-7328. 10.1158/1078-0432.CCR-06-0515

Sung H, Lee KM, Choi JY, Han S, Lee JY, Li L, Park SK, Yoo KY, Noh DY, Ahn SH, and Kang D. 2011. Common genetic polymorphisms of microRNA biogenesis pathway genes and risk of breast cancer: a case-control study in Korea. Breast Cancer Res Treat 130:939-951. 10.1007/s10549-011-1656-2

Thomson JM, Newman M, Parker JS, Morin-Kensicki EM, Wright T, and Hammond SM. 2006. Extensive posttranscriptional regulation of microRNAs and its implications for cancer. Genes Dev 20:2202-2207. 10.1101/gad.1444406

Xie Y, Wang Y, Zhao Y, and Guo Z. 2015. Single-nucleotide polymorphisms of microRNA processing machinery genes are associated with risk for gastric cancer. Onco Targets Ther 8:567-571. 10.2147/OTT.S79150

Yang H, Dinney CP, Ye Y, Zhu Y, Grossman HB, and Wu X. 2008. Evaluation of genetic variants in microRNA-related genes and risk of bladder cancer. Cancer Res 68:2530-2537. 10.1158/0008-5472.can-07-5991

Ye Y, Wang KK, Gu J, Yang H, Lin J, Ajani JA, and Wu X. 2008. Genetic variations in microRNA-related genes are novel susceptibility loci for esophageal cancer risk. Cancer Prev Res (Phila) 1:460-469. 10.1158/1940-6207.CAPR08-0135

Yi R, Qin Y, Macara IG, and Cullen BR. 2003. Exportin-5 mediates the nuclear export of pre-microRNAs and short hairpin RNAs. Genes Dev 17:3011-3016. 10.1101/gad.1158803

Yu YY, Kuang D, and Yin XX. 2015. Association between the DICER rs1057035 polymorphism and cancer risk: evidence from a meta-analysis of 1,2675 individuals. Asian Pac J Cancer Prev 16:119-124.

Yuan L, Chu H, Wang M, Gu X, Shi D, Ma L, Zhong D, Du M, Li P, Tong N, Fu G, Qin C, Yin C, and Zhang Z. 2013. Genetic variation in DROSHA 3'UTR regulated by hsa-miR-27b is associated with bladder cancer risk. PLoS One 8:e81524. 10.1371/journal.pone.0081524

Zhang Q. 2012. Association studies of polymorphisms in miRNA and it's biological processessing genes with the susceptbility of gastric cancer(in chinese). Nanjing medical University.

Zhao Y, Du Y, Zhao S, and Guo Z. 2015. Single-nucleotide polymorphisms of microRNA processing machinery genes and risk of colorectal cancer. Onco Targets Ther 8:421-425. 10.2147/ott.s78647

ZHENG Liang GH-y. 2013. DICER rs3742330A>G ploymorphism and risk of esophageal cancer(in chianese). CHin J Cancer Prec Terat 20:1794-1796.

Zu Y, Ban J, Xia Z, Wang J, Cai Y, Ping W, and Sun W. 2013. Genetic variation in a miR-335 binding site in BIRC5 alters susceptibility to lung cancer in Chinese Han populations. Biochem Biophys Res Commun 430:529-534. 10.1016/j.bbrc.2012.12.001

\section{Supporting Information}

\author{
S1 PRISMA Checklist
}

S2 PROSPERO registration number is: CRD42015024841

S Fig S3: Forest plot of sensitivity analysis. 
377 S Table S4: Characteristics of eligible studies in the meta-analysis.

378 


\section{Table 1 (on next page)}

Characteristics of the studies eligible for meta-analysis. 
1 Table 1. Characteristics of the studies eligible for meta-analysis.

\begin{tabular}{|c|c|c|c|c|c|c|c|c|c|c|}
\hline author & Year & Cancer type & Country & Ethnicity & controls & case & control & method & Polymorphism site & nos \\
\hline Horikawa, Y.[22] & 2008 & renal cell carcinoma & American & Caucasian & PB & 279 & 278 & SNPlex & rs417309,rs13078,rs3742330,rs10719,rs14035 & 8 \\
\hline Yang, $\mathrm{H}^{[23]}$. & 2008 & bladder cancer & American & Caucasian & HB & 746 & 746 & SNPlex & rs417309, s1640299,rs13078,rs3742330,rs1 1077 & 7 \\
\hline Ye, Y.[24] & 2008 & esophageal cancer & American & Caucasian & HB & 346 & 346 & SNPlex & $\begin{array}{c}\text { rs417309,rs1640299,rs13078,rs3742330, } \\
\text { rs14035,rs11077 }\end{array}$ & 7 \\
\hline Kim, J. S. ${ }^{[25]}$ & 2010 & lung cancer & Korea & Asian & HB & 100 & 100 & & $\begin{array}{c}\text { rs417309,rs1640299,rs13078,rs3742330, } \\
\text { rs10719,rs14035 }\end{array}$ & 7 \\
\hline Sung, H. ${ }^{[26]}$ & 2011 & breast cancer & Korea & Asian & HB & 559 & 567 & TaqMan & rs1057035,rs11077 & 7 \\
\hline $\mathrm{Ma}, \mathrm{H}^{[27]}$ & 2012 & head and neck cancer & China & Asian & HB & 397 & 900 & TaqMan & rs $1057035, \mathrm{rs} 3803012$ & 7 \\
\hline Chen, J.[28] & 2013 & cervical carcinoma & China & Asian & HB & 1486 & 1549 & TaqMan & rs1057035,rs3803012 & 7 \\
\hline Jiang, $Y^{[29]}$ & 2013 & breast cancer & China & Asian & HB & 878 & 900 & TaqMan & $\begin{array}{c}\text { rs417309,rs1640299,rs1057035,rs13078, } \\
\text { rs10719,rs3803012 }\end{array}$ & 7 \\
\hline Liu, L. ${ }^{[30]}$ & 2013 & hepatocellular carcinoma & China & Asian & HB & 1300 & 2688 & TaqMan & rs417309, rs164029,rs1057035,rs3803012 & 7 \\
\hline Slaby, $\mathrm{O}^{[31]}$ & 2013 & colorectal cancer & Czech & Caucasian & HB & 197 & 202 & TLDA & rs 1057035 & 7 \\
\hline Yuan, L. ${ }^{[32]}$ & 2013 & bladder cancer & China & Asian & HB & 685 & 730 & TaqMan & rs1057035,rs13078,rs3742330,rs10719 & 7 \\
\hline Roy, Roshni ${ }^{[33]}$ & 2014 & oral cancer & India & Asian & HB & 451 & 452 & Taqman & rs14035 & 7 \\
\hline Cho, S. H. ${ }^{[34]}$ & 2015 & Colorectal Cancer & Korean & Asian & $\mathrm{HB}$ & 408 & 400 & PCR-RFLP & rs3742330,rs10719,rs14035,rs11077 & 7 \\
\hline Martin-Guerrero, I. ${ }^{[35]}$ & 2015 & Lymphocytic Leukemia & Spanish & Caucasian & HB & 123 & 391 & Taqman & $\begin{array}{l}\text { rs417309,rs1640299,rs1057035,rs13078, } \\
\text { rs10719,rs14035 }\end{array}$ & 7 \\
\hline Xie, Y. ${ }^{[36]}$ & 2015 & gastric cancer & China & Asian & HB & 137 & 142 & & rs3742330,rs14035,rs11077 & 7 \\
\hline Zhao, Y. ${ }^{[37]}$ & 2015 & colorectal cancer & China & Asian & $\mathrm{HB}$ & 163 & 142 & & rs3742330,rs14035,rs11077 & 7 \\
\hline
\end{tabular}




\begin{tabular}{|l|c|c|c|c|c|c|c|c|c|c|}
\hline Zu, Y. ${ }^{[38]}$ & 2013 & lung cancer & China & Asian & HB & 600 & 600 & TaqMan & 7 \\
\hline Zheng Liang. ${ }^{[39]}$ & 2013 & esophageal cancer & China & Asian & HB & 380 & 380 & & rs1057035, & rs3742330 \\
\hline Zhang Qin ${ }^{[40]}$ & 2012 & gastric cancer & China & Asian & HB & 1674 & 1852 & TaqMan & rs3803012,rs1057035 & 7 \\
\hline Buas, M. F.[41] & 2015 & esophageal cancer & Europe & Caucasian & HB & 600 & 600 & TaqMan & rs 14035, rs 11077 \\
\hline Gutierrez-Camino, A. ${ }^{[42]}$ & 2014 & Lymphocytic Leukemia & Spanish & Caucasian & HB & 213 & 387 & & & 7 \\
\hline
\end{tabular}


Table 2 (on next page)

Analysis of associations between SNPs from DROSHA, DGCR8, XPO5, RAN, and DICER1 and cancer risk.

*W: major allele M: minor allele a: Asian population b: Caucasian population c: all over \#: WM+MM vs. WW Method: F: Fixed model R: Random model 
1 Table 2. Analysis of associations between SNPs from DROSHA, DGCR8, XPO5, RAN, and DICER1 and cancer risk.

\begin{tabular}{|c|c|c|c|c|c|c|c|c|c|c|c|c|c|}
\hline \multirow{2}{*}{ Gene(locus) } & \multirow{2}{*}{ Position } & \multirow{2}{*}{ studies } & \multirow{2}{*}{ Method } & \multirow{2}{*}{ Cases/controls } & WW vs.WM+MM & \multirow{2}{*}{$P^{a}$} & \multirow{2}{*}{$I^{2}$} & WW vs. WM+MM & \multirow{2}{*}{$P^{b}$} & \multirow{2}{*}{$I^{2}$} & WW vs.WM+MM & \multirow{2}{*}{$P^{c}$} & \multirow{2}{*}{$I^{2}$} \\
\hline & & & & & $\mathrm{OR}^{\mathrm{a}}(\mathbf{9 5 \%} \mathrm{CI})$ & & & $\mathrm{OR}^{\mathrm{b}}(95 \% \mathrm{CI})$ & & & $\mathrm{OR}^{\mathrm{c}}(95 \% \mathrm{CI})$ & & \\
\hline \multicolumn{14}{|l|}{ DROSHA(5p13.3) } \\
\hline $\mathrm{rs} 10719 \mathrm{~T}>\mathrm{C}$ & 3'UTR & 5 & $\mathrm{R}$ & $1982 / 2293$ & $0.91(0.75,1.10)$ & 0.209 & 36.2 & $1.34(0.78,2.30)$ & 0.070 & 69.6 & $1.05(0.83,1.33)$ & 0.026 & 63.9 \\
\hline \multicolumn{14}{|l|}{$D G C R 8(22 \mathrm{q} 11.2)$} \\
\hline rs $417309 \mathrm{G}>\mathrm{A} \#$ & 3'UTR & 7 & $\mathrm{~F}$ & $3327 / 3658$ & $1.44(1.13,1.83)$ & 0.561 & 0.0 & $1.04(0.78,1.39)$ & 0.147 & 47.8 & $1.22(1.04,1.42)$ & 0.190 & 31.2 \\
\hline rs $1640299 \mathrm{~T}>\mathrm{G}$ & 3'UTR & 7 & $\mathrm{R}$ & $2610 / 3046$ & $1.07(0.90,1.28)$ & 0.571 & 0.0 & $1.22(0.85,1.75)$ & 0.017 & 70.7 & $1.20(0.94,1.54)$ & 0.038 & 46.1 \\
\hline \multicolumn{14}{|l|}{ XPO5(6p21.1) } \\
\hline $\mathrm{rs} 11077 \mathrm{~A}>\mathrm{G}$ & 3'UTR & 7 & $\mathrm{~F}$ & $8065 / 5478$ & $0.83(0.66,1.03)$ & 0.652 & 0.0 & $0.95(0.88,1.03)$ & 0.260 & 25.7 & $0.94(0.87,1.01)$ & 0.463 & 0.0 \\
\hline \multicolumn{14}{|l|}{$R A N(12 \mathrm{q} 24.3)$} \\
\hline rs $14035 \mathrm{C}>\mathrm{T}$ & 3'UTR & 9 & $\mathrm{R}$ & $7702 / 5335$ & $1.40(0.72,2.69)$ & 0.001 & 92.6 & $0.99(0.73,1.33)$ & 0.002 & 79.2 & $1.17(0.87,1.57)$ & 0.000 & 88.7 \\
\hline $\mathrm{rs} 3803012 \mathrm{~A}>\mathrm{G}$ & 3'UTR & 5 & F & $5642 / 6489$ & $0.98(0.87,1.11)$ & 0.494 & 0.0 & - & - & - & - & - & - \\
\hline \multicolumn{14}{|l|}{ DICERI(14q32.13) } \\
\hline $\mathrm{rs} 1057035 \mathrm{~T}>\mathrm{C}$ & 3'UTR & 10 & F & $7783 / 8925$ & $1.13(1.04,1.22)$ & 0.163 & 33.2 & $1.21(0.90,1.62)$ & 0.182 & 44.0 & $1.13(1.05,1.22)$ & 0.188 & 27.8 \\
\hline $\mathrm{rs} 3742330 \mathrm{~A}>\mathrm{G}$ & 3'UTR & 9 & $\mathrm{R}$ & $3222 / 3240$ & $1.02(0.89,1.18)$ & 0.000 & 79.6 & $0.90(0.73,1.10)$ & 0.396 & 0.0 & $0.98(0.87,1.10)$ & 0.001 & 70.9 \\
\hline $\mathrm{rs} 13078 \mathrm{~T}>\mathrm{A}$ & 3'UTR & 7 & $\mathrm{~F}$ & $3102 / 3419$ & $1.01(0.81,1.26)$ & 0.889 & 0.0 & $1.13(0.97,1.32)$ & 0.116 & 49.3 & $1.09(0.96,1.24)$ & 0.339 & 11.8 \\
\hline
\end{tabular}




\section{Table 3 (on next page)}

Table 3. Results of Egger's and Begg's tests for publication bias. 
1 Table 3. Results of Egger's and Begg's tests for publication bias.

\begin{tabular}{|c|c|c|c|c|c|}
\hline \multirow{2}{*}{ Category } & \multirow{2}{*}{ Studies } & \multicolumn{2}{|c|}{ Begg's test } & \multicolumn{2}{|c|}{ Egger's test } \\
\hline & & $\mathbf{Z}$ & $P$-value & $(95 \%) \mathrm{CI}$ & $P$-value \\
\hline \multicolumn{6}{|l|}{ DROSHA } \\
\hline rs10719T $>C$ & 5 & 1.47 & 0.14 & $(-0.87,7.07)$ & 0.09 \\
\hline \multicolumn{6}{|l|}{$D G C R 8$} \\
\hline rs417309G $>A$ & 7 & 1.20 & 0.23 & $(-4.79,0.79)$ & 0.13 \\
\hline rs1640299T $>G$ & 7 & 1.20 & 0.23 & $(-2.39,5.13)$ & 0.39 \\
\hline \multicolumn{6}{|l|}{ XPO5 } \\
\hline rs11077A $>G$ & 7 & 0.3 & 0.76 & $(-0.20,0.12)$ & 0.53 \\
\hline \multicolumn{6}{|l|}{$R A N$} \\
\hline rs $14035 \mathrm{C}>\mathrm{T}$ & 9 & 1.15 & 0.25 & $(-2.66,5.07)$ & 0.49 \\
\hline $\mathrm{rs} 3803012 \mathrm{~A}>\mathrm{G}$ & 5 & -1.32 & 0.19 & $(-3.75,1.80)$ & 0.39 \\
\hline \multicolumn{6}{|l|}{ DICER1 } \\
\hline rs $1057035 \mathrm{~T}>\mathrm{C}$ & 10 & 0.72 & 0.47 & $(-2.11,4.20)$ & 0.76 \\
\hline rs 3742330A $>$ G & 9 & 0.10 & 0.92 & $(-2.98,8.01)$ & 0.32 \\
\hline rs13078T $>A$ & 7 & -1.05 & 0.293 & $(-3.81,2,91)$ & 0.77 \\
\hline
\end{tabular}

2 
Figure 1 (on next page)

MiRNA biogenesis pathway. 
Figure 2 (on next page)

Flow diagram of the study selection process. 


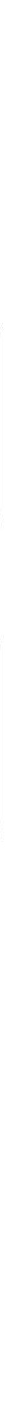




\section{Figure 3 (on next page)}

Figure 3: Forest plot for the relationship between the microRNA biogenesis pathway genes polymorphism and cancer risk $\square$ 


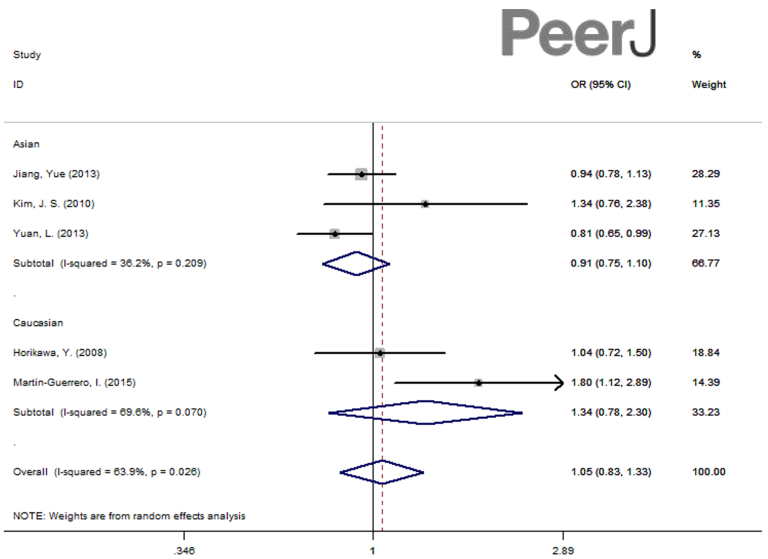

A:rs10719T $>$ C

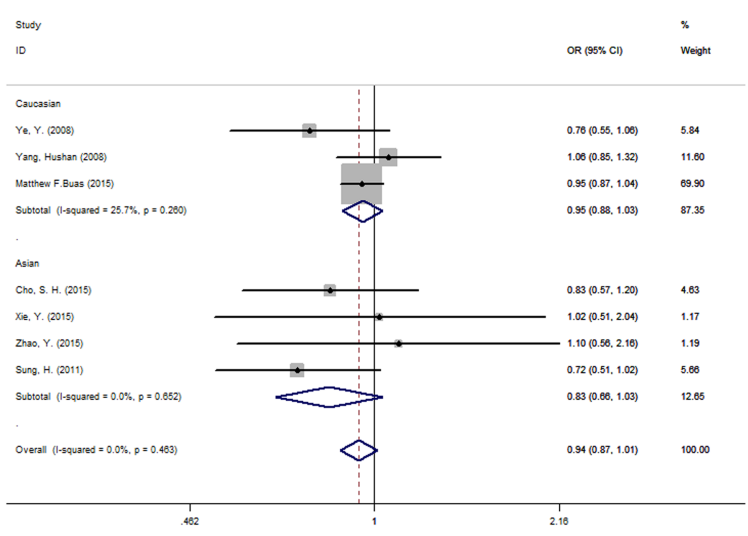

D:rs $11077 \mathrm{~A}>\mathrm{G}$

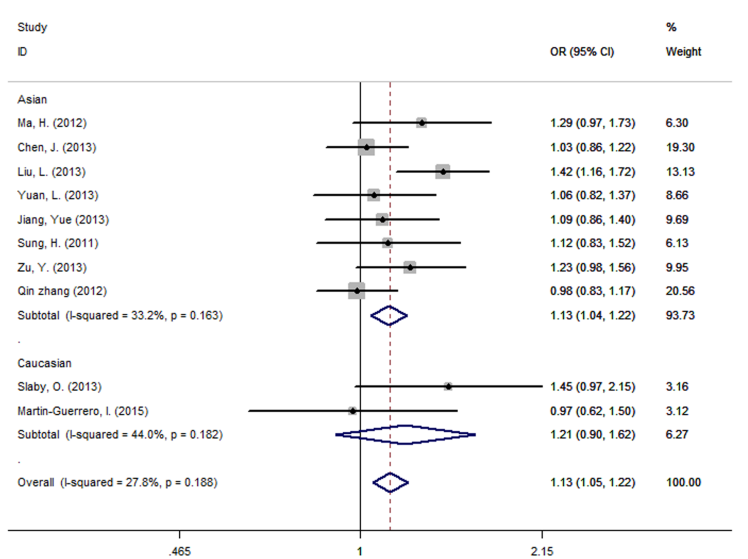

Manusçipt to be reviewed

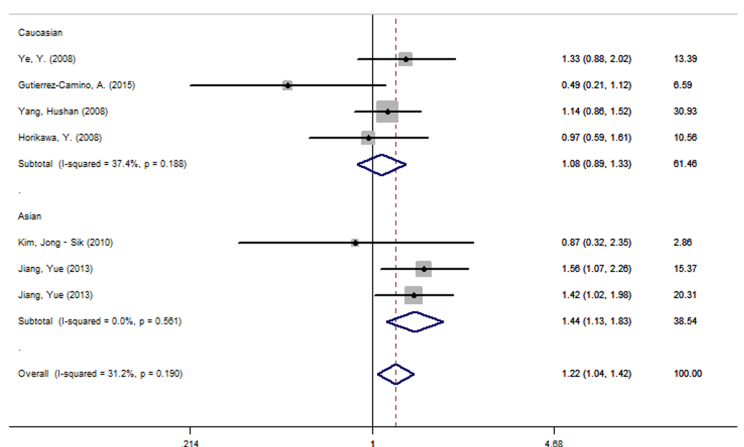

B:rs417309G $>$ A

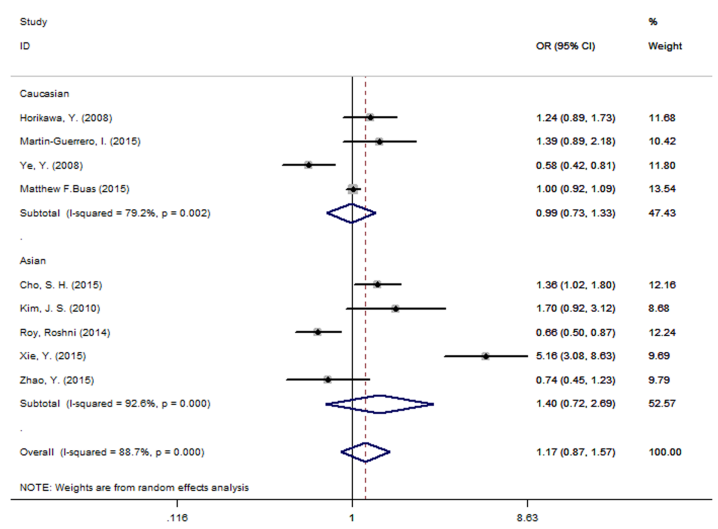

E:rs $14035 \mathrm{C}>\mathrm{T}$

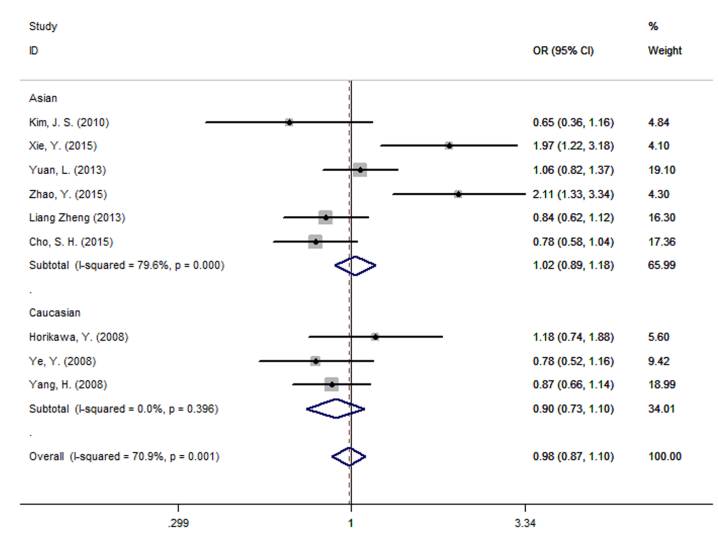

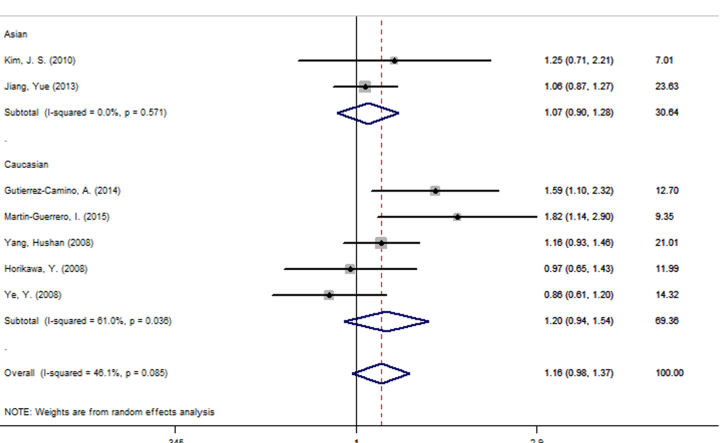

C: $\operatorname{rs} 1640299 \mathrm{~T}>\mathrm{G}$

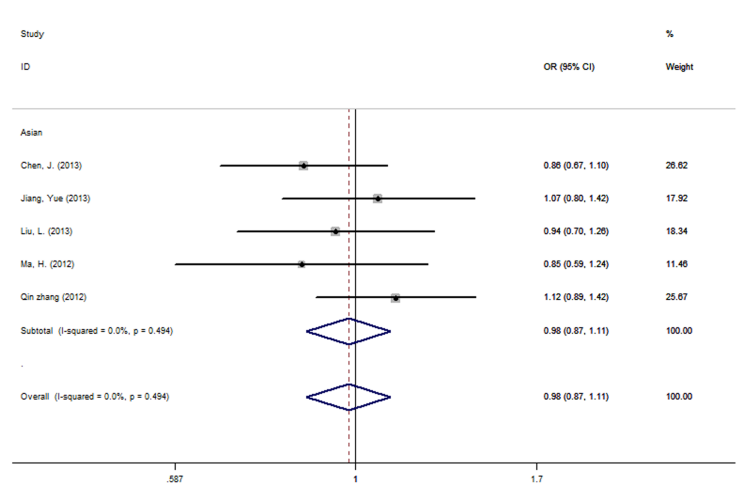

F: rs3803012A $>\mathrm{G}$

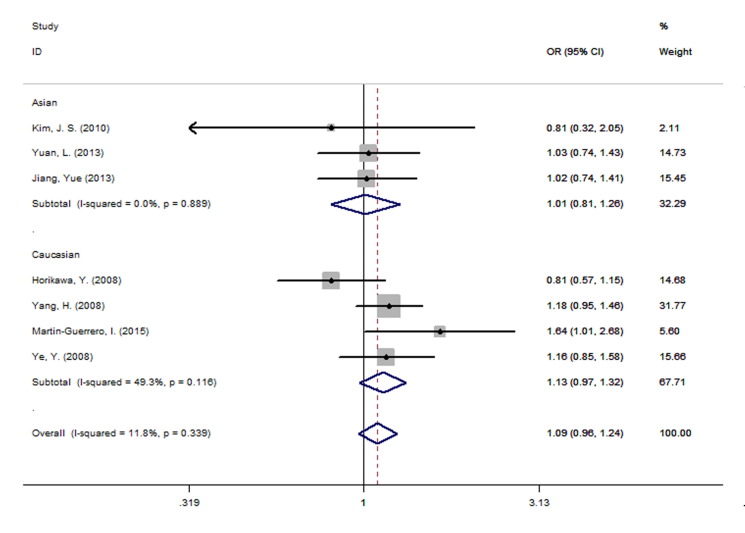

I:rs13078T $>$ A 
Figure 4 (on next page)

Fig 4: Funnel plot for publication bias test. $\mathrm{OR}=$ odds ratio, $\mathrm{SE}=$ standard error 


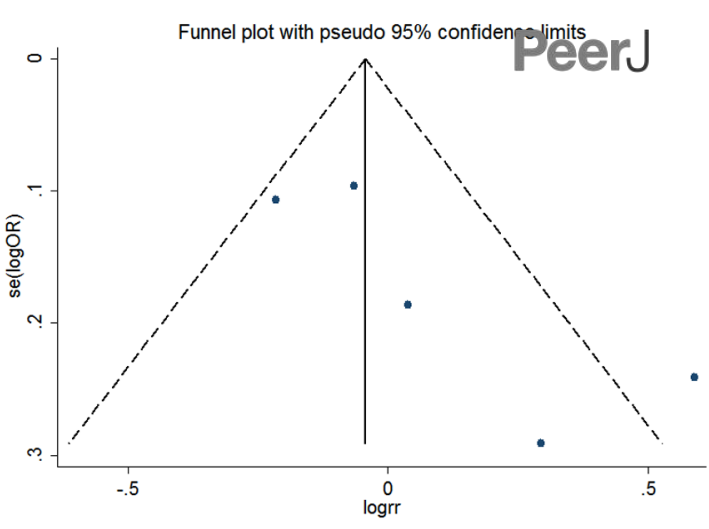

A:rs10719T $>C$

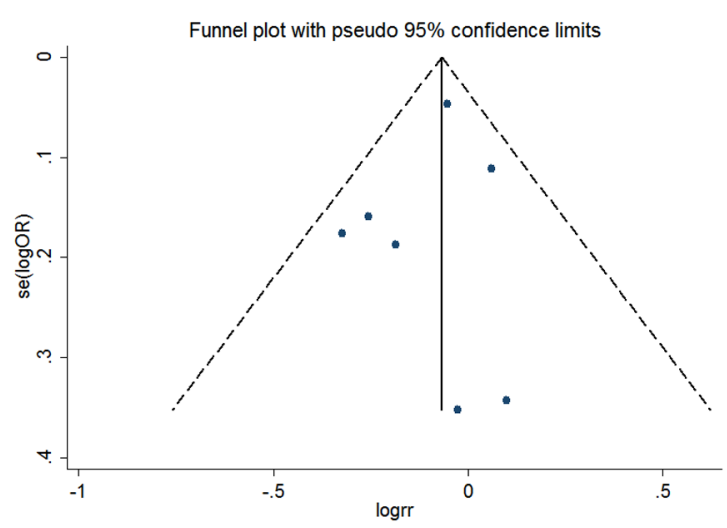

D:rs 11077A >G

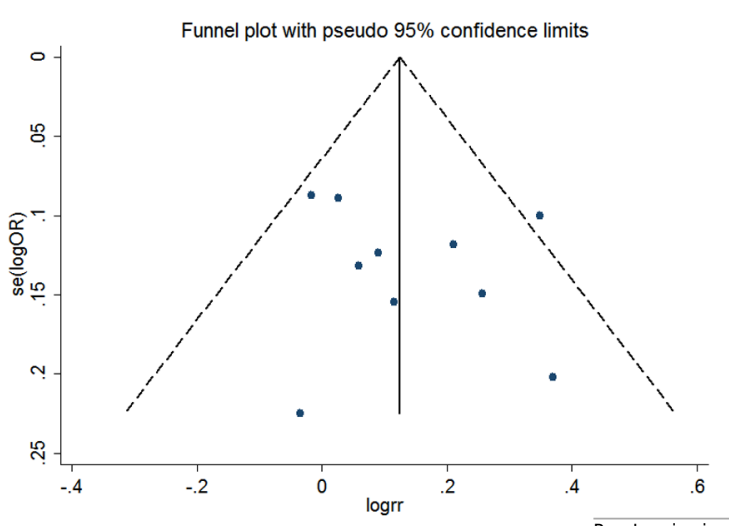

G:rs $1057035 T>C$

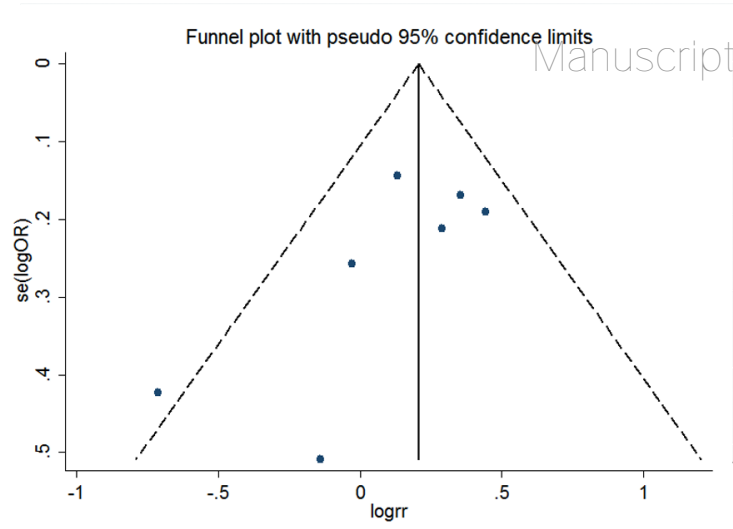

B:rs417309G $>A$

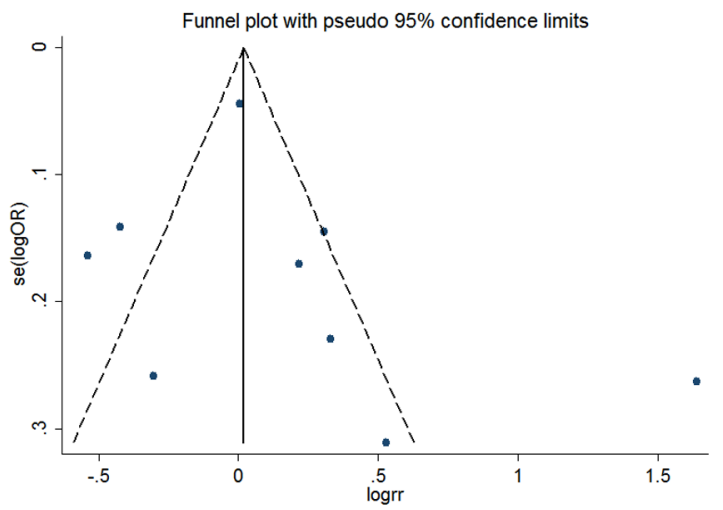

E:rs14035C $>$ T

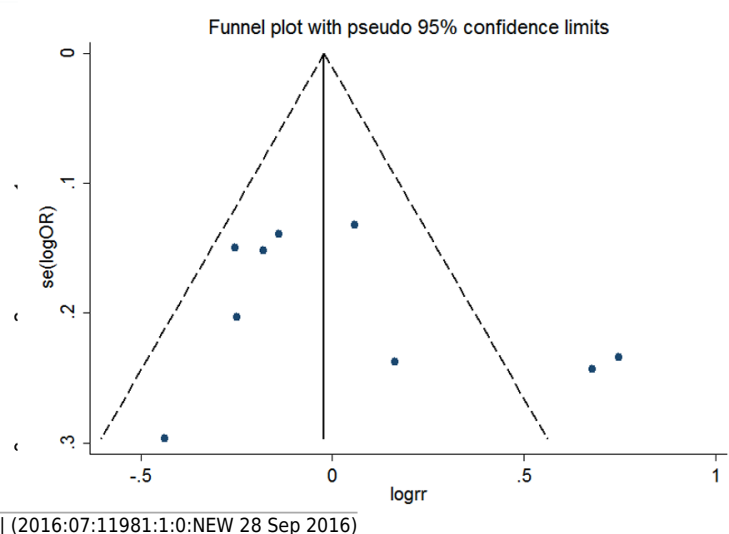

H:rs3742330A $>G$ be review ed Funnel plot with pseudo 95\% confidence limits

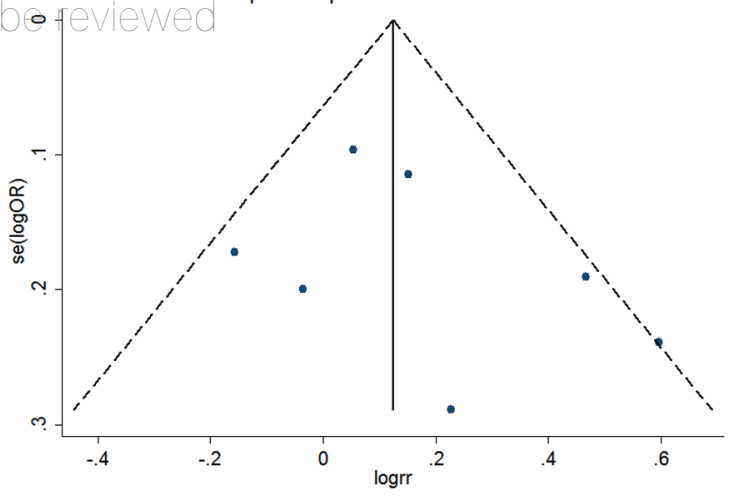

C: $\operatorname{rs} 1640299 \mathrm{~T}>\mathrm{G}$

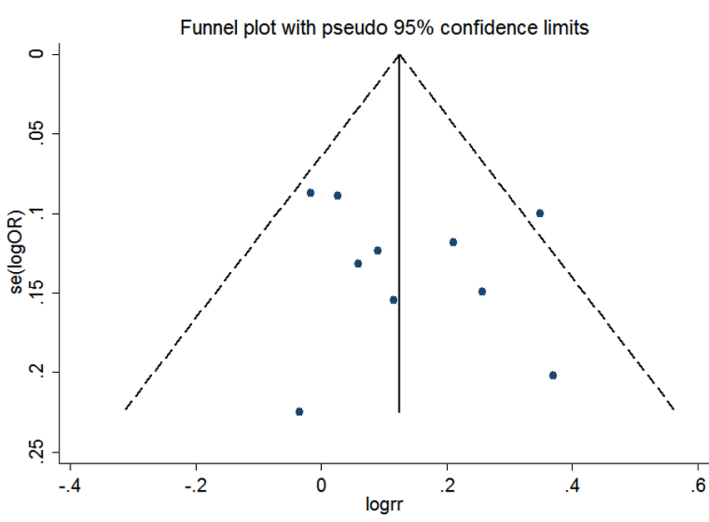

F: $r s 3803012 A>G$

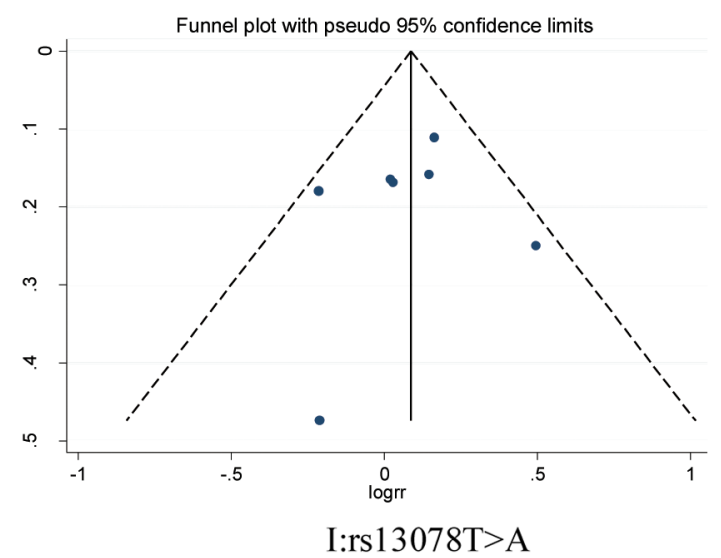

